\title{
Effects of oral amino acid supplementation on Multidimensional Prognostic Index in hospitalized older patients: a multicenter randomized, double- blind, placebo-controlled pilot study
}

This article was published in the following Dove Press journal:

Clinical Interventions in Aging

\section{Stefano Volpato' \\ Romina Custureri ${ }^{2}$ \\ Matteo Puntoni ${ }^{3}$ \\ Lara Bianchi' \\ Julia Daragjati ${ }^{4}$ \\ Sara Garaboldi ${ }^{2}$ \\ Matteo Simonato ${ }^{4}$ \\ Antonio Greco 5 \\ Emanuele Rizzo 6 \\ Pierluigi Dal Santo ${ }^{7}$ \\ Antonio Mangiacotti ${ }^{5}$ \\ Ekaterini Zigoura ${ }^{2}$ \\ Giacomo Siri ${ }^{3}$ \\ Alberto Pilotto ${ }^{2}$ \\ 'Department of Medical Science, University of Ferrara, Ferrara, Italy; ${ }^{2}$ Department of Geriatric Care, Orthogeriatrics and Rehabilitation, E.O. Galliera Hospital, Genova, Italy; ${ }^{3}$ Clinical Trial Unit, Scientific Directorate, E.O. Galliera Hospital, Genoa, Italy; ${ }^{4}$ Geriatrics Unit, San Antonio Hospital, ULSS 6 Euganea, Padova, Italy; ${ }^{5}$ Geriatrics Unit, IRCCS Casa Sollievo della Sofferenza, San Giovanni Rotondo, Foggia, Italy; ${ }^{6}$ Geriatrics Unit, ULSS 2 Marca Trevigiana, Montebelluna, Italy; ${ }^{7}$ Geriatrics Unit, ULSS 5 Polesana, Rovigo, Italy}

Correspondence: Stefano Volpato Department of Medical Science, University of Ferrara, Via Ariosto, 35 I-44 I 2 I Ferrara, Italy

Tel +390532 236658

Fax +390532 210 884

Email vlt@unife.it
Background: It is not known whether amino acid supplementations may influence health status in hospitalized older acutely ill patients.

Aim: The aim of this study was to determine whether nutritional supplementation with amino acids $\left(\right.$ Aminoglutam $\left.^{\circledR}\right)$ is associated with multidimensional improvement assessed with the Multidimensional Prognostic Index (MPI).

Methods: In this randomized, double-blind, placebo-controlled pilot clinical trial, 126 patients aged $\geq 65$ years were enrolled from 6 Italian geriatric wards. A multidimensional assessment to calculate the MPI was performed at baseline and after 4 weeks of treatment with nutritional supplementation ( $96 \mathrm{kcal}, 12 \mathrm{~g}$ amino acids, $0.18 \mathrm{~g}$ fat, $11.6 \mathrm{~g}$ carbohydrate, and vitamins $\mathrm{B} 1$, B6, and C) or placebo administered twice a day. Logistic regression modeling was applied to determine the effect of treatment on the improvement of MPI (vs no-change/worsening), adjusting for gender, age, and MPI at baseline. Treatment's interactions with age, gender, and MPI at baseline were tested adding the appropriate interaction parameter in the regression models.

Results: Of the 126 patients included, 117 patients (93\%) completed the study. A significant improvement in the MPI score was detected in the overall population (mean difference postpretreatment: $-0.03, p=0.001$ ), with no differences between active and placebo arms. Men in the amino acid supplementation group had a significantly higher rate of improvement in MPI (81\%) compared to the placebo group (46\%) (Fisher's exact test $p=0.03$ ). Adjusting for age, diagnosis, and MPI at baseline, amino acid treatment was shown to be associated with an improvement in MPI in men (OR=4.82, 95\% confidence interval [CI]: 0.87-26.7) and not in women $(\mathrm{OR}=0.70,95 \% \mathrm{CI}: 0.27-1.81)$. The interaction effect between active treatment and gender was significant $(p=0.04)$.

Conclusion: A 4-week amino acid supplementation improved the MPI significantly in hospitalized older male patients but not in female patients. Further studies are needed to confirm the gender effect of amino acid supplementation on MPI in older patients.

Keywords: Multidimensional Prognostic Index, aging, hospitalization, oral supplementation, amino acids

\section{Introduction}

Hospitalization for an acute medical event represents a stressful and potentially hazardous event for older persons, which often leads to important clinical complications, including functional decline, prolonged length of stay, and death. ${ }^{1}$ Besides the disabling effect of the acute event, hospitalization itself might represent an additional 
stressor in terms of environmental hazard, reduced caloric intake, low physical activity or prolonged bed rest, depressed mood, and social isolation. ${ }^{2}$ For example, in older patients, hospitalization and prolonged bed rest have been associated with increased risk of incident sarcopenia and functional decline. ${ }^{3}$

Malnutrition, and specifically energy-protein malnutrition, is common in older patients, and hospitalized patients are often at risk of malnutrition during hospital stay. ${ }^{4}$ An optimal amino acid availability not only is fundamental to guarantee an adequate protein synthesis but also plays a crucial role in several conditions characterized by a hypercatabolic state including many acute diseases, cancers, and inflammatory conditions. ${ }^{5}$ Malnutrition is associated with poor health status and poor clinical outcomes in hospitalized adults, including prolonged hospital stay, increased risk of hospital readmission, and increased mortality. ${ }^{6}$

Several studies investigated the effect of oral nutritional supplementation on nutritional status and clinical outcomes in older patients admitted to the hospital. Globally taken, these studies suggest that oral supplementation increased nutritional status, including body weight, but the effect on more relevant clinical outcomes is still controversial. ${ }^{7}$

This pilot study evaluated the effect of a nutritional supplement containing $12 \mathrm{~g}$ of amino acids on post-discharge health status, assessed using the Multidimensional Prognostic Index (MPI). MPI is a prognostic instrument based on 6 commonly used geriatric assessment scales exploring cognitive, functional, nutritional, and clinical status, as well as on information about drugs taken and patient's social support. ${ }^{8}$ MPI is an accurate index to assess mortality risk and overall health status of older people; in older hospitalized patients, MPI predicts length of hospital stay, in-hospital mortality, and long-term mortality after hospital discharge. ${ }^{9,10} \mathrm{We}$ hypothesized that compared to patients allocated to placebo, those randomized to amino acid supplementation significantly improve the MPI score over the follow-up.

\section{Methods Study design}

The Frailty and Amino acid supplementation (FRAMINO) study was a pilot multicenter, prospective, randomized, double-blind, placebo-controlled, parallel group study conducted in Italy between June 1, 2014, and January 31, 2016. The study evaluated the effects of Aminoglutam ${ }^{\circledR}$ (AG) vs placebo on the changes in the overall health status in older hospitalized adults. As per the initial protocol, change in the MPI score at 30 days after randomization was the primary outcome. Patient assessments were performed at baseline and 30 days after randomization or at study discontinuation and included the same clinical evaluation performed at baseline. The protocol received approval from the ethical committee of the coordinating center (Comitato Etico IRCCS "Casa Sollievo della Sofferenza" - San Giovanni Rotondo, Foggia) and from ethical committees of all other sites (Comitato Etico Azienda ULSS 16 - Padova - Nucleo per la Ricerca clinica; Comitato Etico unico della provincia di Ferrara; Comitato Etico Regionale [CER] - Regione Liguria; Comitato Etico per la Sperimentazione Clinica della Provincia di Rovigo; and Comitato Etico per la sperimentazione clinica delle province di Treviso e Belluno). All patients provided written informed consent.

\section{Patients}

All patients aged 65 years or more consecutively admitted to participating hospital wards entered the study protocol regardless of the diagnosis of admission. Eligible patients were required to have an MPI grade 2 or 3 (expression of moderate or severe mortality risk, respectively). Exclusion criteria were current active advanced cancer or severe organ failure that might prevent the 30-day follow-up evaluation.

MPI is a validated prognostic index based on a Comprehensive Geriatric Assessment (CGA) that accurately predicts short- and long-term mortality in older people in different clinical settings and affected by different medical conditions. To calculate the MPI, a standardized CGA was performed within 48 hours from admission to collect information on basic activities of daily living (ADL) and instrumental activities of daily living (IADL) according to the Katz et $\mathrm{al}^{11}$ and the Lawton-Brody ${ }^{12}$ scales, respectively. Cognitive status was evaluated using the Short Portable Mental Status Questionnaire (SPMSQ). ${ }^{13}$ Comorbidity burden was evaluated using the comorbidity subscale of the cumulative illness rating scale (CIRS), ${ }^{14}$ and nutritional status was assessed through the mini nutritional assessment (MNA). ${ }^{15}$ The Exton-Smith scale (ESS) was used to evaluate the risk of developing pressure ulcers. ${ }^{16}$ The number of medications taken at home and co-habitation status (alone, in institution, and with family) was recorded. All site personnel were trained on MPI administration and evaluation.

\section{Study treatments}

After eligibility criteria were ascertained, patients were randomized (1:1) through a centralized allocation system to receive either AG or placebo twice daily during hospitalization and 30 days after randomization. After randomization, 
both groups of patients continued the pharmacological and/or dietetic prescription indicated by the attending physician, and after hospital discharge they continued that prescribed by the primary care physician. AG is a specialized, dietetic aliment (Errekappa Euroterapici S.p.a., Milan, Italy) with $96 \mathrm{kcal}, 12 \mathrm{~g}$ amino acids (including essential amino acids), $0.18 \mathrm{~g}$ fat, $11.6 \mathrm{~g}$ carbohydrate, and vitamins B1, B6, and C. Placebo and AG formula share only excipients, intended as not therapeutic agents. The detailed nutritional composition of AG is presented in Table S1.

\section{Outcomes}

The primary outcome for the study was change in the MPI score at the end of the 4-week follow-up period. Other efficacy end points included change in distribution of the MPI group and likelihood of MPI improvement over the follow-up. As pre-specified in the study protocol, secondary analyses were stratified according to the baseline MPI group and gender.

\section{Statistical analysis}

Efficacy analyses were performed on the intention-to-treat (ITT) population, defined a priori as all enrolled patients who received any amount of allocated intervention. The sample size was calculated based on the 30-day change in the MPI score. With a sample size of 60 patients per arm, the study was $95 \%$ powered to detect a MPI change difference of $\geq 0.07$ in the active arm, assuming no change in the placebo arm. The sample size calculation was performed assuming an SD of the MPI difference equal to 0.15 (previous studies ${ }^{27}$ ) and a 0.01 2-sided alpha level.
Demographics and clinical characteristics were summarized using mean and SD for continuous factors and absolute and relative $(\%)$ frequencies for categorical factors. Pearson chi-square or Fisher's exact tests and independent sample $t$-test were adopted to compare categorical and continuous variables, respectively, between arms. Changes in primary (MPI) and component efficacy end points according to the treatments groups were analyzed and compared using the paired $t$-test and ANCOVA, setting the change in MPI as a dependent variable and treatment as explanatory, adjusting for main demographics and clinical factors. Logistic regression analysis was adopted to investigate the likelihood of MPI improvement (vs no change or worsening) at the end of the follow-up as a function of treatment group, adjusting for age and MPI at baseline.

\section{Results \\ Patients}

A total of 126 patients were enrolled from 6 sites (62 AG and 64 placebo) and are included in the ITT population; 9 patients (2 AG and 7 placebo) exited the study prior to the 30-day follow-up visit: 5 because of side effects and 4 because they were lost to follow-up (Figure 1). Overall, clinical outcomes were unavailable from 2 and 7 patients in the AG and placebo groups, respectively. Demographics, clinical characteristics, and conditions leading to hospital admission were comparable between groups, except for the prevalence of patients malnourished or at risk of malnutrition (MNA score <24), which was greater in the active intervention group (Table 1). Patients randomized to placebo were more likely to be male

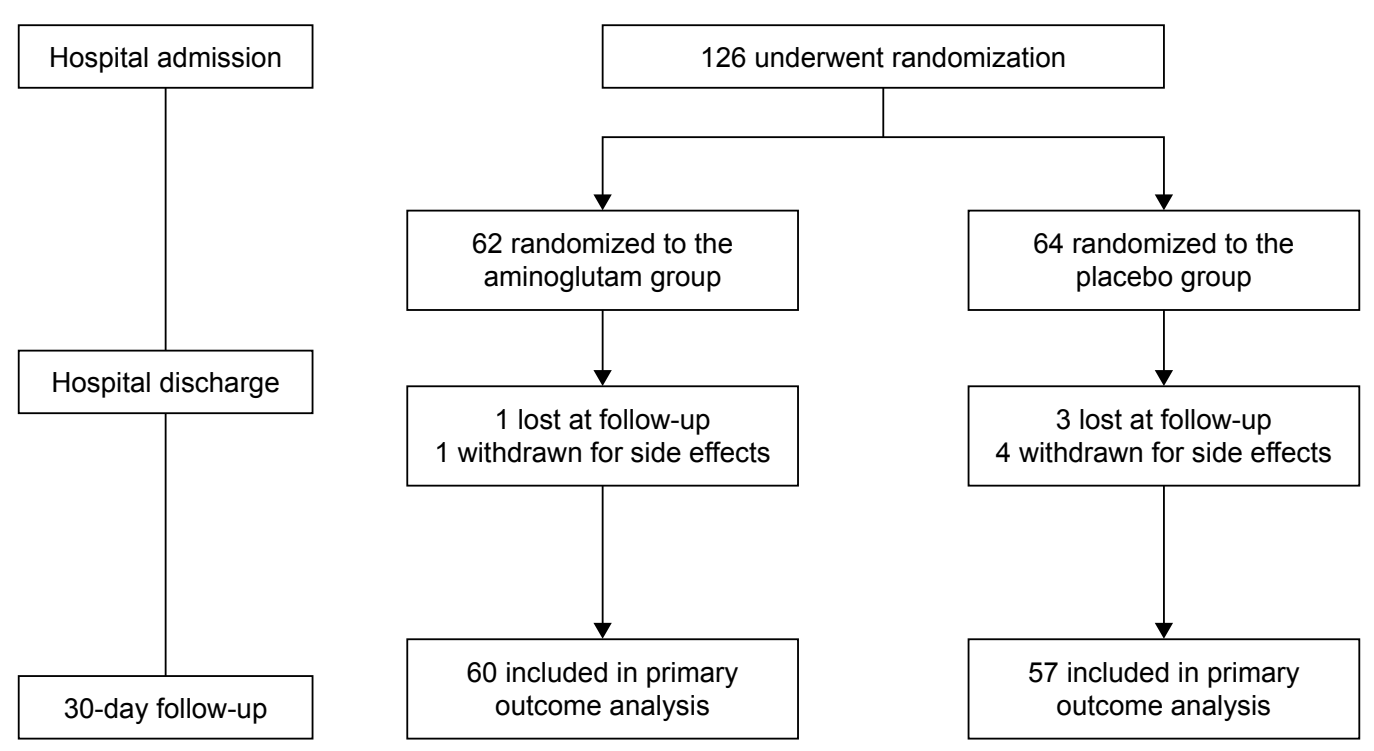

Figure I Study design and number of patients enrolled and included in the analysis. 
Table I Baseline selected demographic and clinical characteristics of the treatment groups

\begin{tabular}{|c|c|c|c|}
\hline Characteristics & $\begin{array}{l}\text { Active } \\
(n=62)\end{array}$ & $\begin{array}{l}\text { Placebo } \\
(n=64)\end{array}$ & $p$-value \\
\hline Age, years mean $( \pm S D)$ & $84.5(6.0)$ & $84.7(5.6)$ & 0.792 \\
\hline Gender $(M / F, \%$ of $M)$ & I7/45, 27.4 & $25 / 39,39.1$ & 0.189 \\
\hline $\mathrm{BMI}, \mathrm{kg} / \mathrm{m}^{2}$ mean $( \pm \mathrm{SD})$ & $23.2(3.2)$ & $23.4(4.1)$ & 0.805 \\
\hline Calf circumference, $\mathrm{cm}$ mean $( \pm \mathrm{SD})$ & $30.9(2.7)$ & $31.8(2.6)$ & 0.074 \\
\hline \multicolumn{4}{|l|}{ Primary admission conditions, $\mathrm{n}(\%)$} \\
\hline Cardiovascular & $24(38.7)$ & $19(29.7)$ & 0.348 \\
\hline Pulmonary & $10(16.1)$ & $12(18.8)$ & 0.816 \\
\hline Neurological & $5(8.1)$ & $6(9.4)$ & I \\
\hline Gastroenterological & $4(6.5)$ & $4(6.3)$ & I \\
\hline Osteoarticular & $4(6.5)$ & $3(4.7)$ & $0.7 \mid 5$ \\
\hline Cerebrovascular diseases & $2(3.2)$ & $3(4.7)$ & 1.000 \\
\hline Sepsis & $4(6.5)$ & $0(-)$ & 0.056 \\
\hline Others & $9(14.5)$ & $17(26.6)$ & 0.124 \\
\hline MPI mean $( \pm S D)$ & $0.57(0.16)$ & $0.54(0.17)$ & 0.275 \\
\hline SPMSQ & $3.58(2.85)$ & $3.20(2.90)$ & 0.463 \\
\hline Exton-Smith scale & $14.58(2.8 I)$ & $14.97(3.20)$ & 0.471 \\
\hline ADL & $2.79(2.00)$ & $3.38(2.23)$ & 0.124 \\
\hline IADL & $2.66(2.25)$ & $2.83(2.24)$ & 0.677 \\
\hline CIRS & $3.89(2.04)$ & $3.80(1.80)$ & 0.793 \\
\hline MNA & $17.48(5.12)$ & $|8.5|(4.79)$ & 0.246 \\
\hline MNA score $<24, * N(\%)$ & 57 (91.9) & $50(78.1)$ & 0.036 \\
\hline Number of drugs & $6.47(2.49)$ & $6.42(2.53)$ & 0.919 \\
\hline
\end{tabular}

Note: $* M N A$ score $<24$ : malnourished or at risk of malnutrition.

Abbreviations: ADL, activities of daily living; BMI, body mass index; CIRS, cumulative illness rating scale; $F$, female; IADL, instrumental activities of daily living; M, male; MNA, mini nutritional assessment; MPI, Multidimensional Prognostic Index; SPMSQ, Short Portable Mental State Questionnaire.

and had more ADL impairment, but the differences were not statistically significant. Acute cardiovascular disease (34\%) and acute pulmonary conditions (17.4\%) were the most common causes of hospitalization.

\section{Efficacy end points}

At the 30-day follow-up visit, the MPI score significantly decreased in the overall sample $(-0.03,95 \%$ confidence interval $[\mathrm{CI}]-0.05$ to -0.01 ) without significant differences between study groups, suggesting an overall improvement of the health status of the study population over time, independently of AG treatment (Figure 2).

Analysis of single components of MPI (Table 2) demonstrated similar difference between treatment groups; the placebo group experienced a greater increase in the ESS score compared to the AG group, although the difference was only of borderline statistical significance ( $p=0.07$ ).

The MPI score improved in approximately $50 \%$ of patients at day 30 without significant difference between groups (51.7 and $47.4 \%$ in the $\mathrm{AG}$ and placebo groups, respectively) (Figure 3). However, men in AG supplementation group had a significantly higher rate of improvement compared to the placebo counterpart ( 81.3 vs $45.8 \%, p=0.03$ ). After adjustment for age and baseline MPI score, we found a significant interaction between gender and treatment $(p=0.05)$, suggesting that the effect of amino acid supplementation on MPI change is different in men compared to women.

\section{Safety}

A very small proportion of patients $(5,3.9 \%$ of the study population) reported treatment adverse events, 1 in the AG group and 4 in the placebo group, respectively (Fisher's exact test: 0.365$)$. The reported adverse events were headache (1 patient in AG), diarrhea (3 cases in the placebo group), and vomiting (1 patient in the placebo group).

\section{Discussion}

Decline in health and functional status is common in older patients admitted to the hospital for an acute medical event, and sarcopenia is often associated with both hospitalization

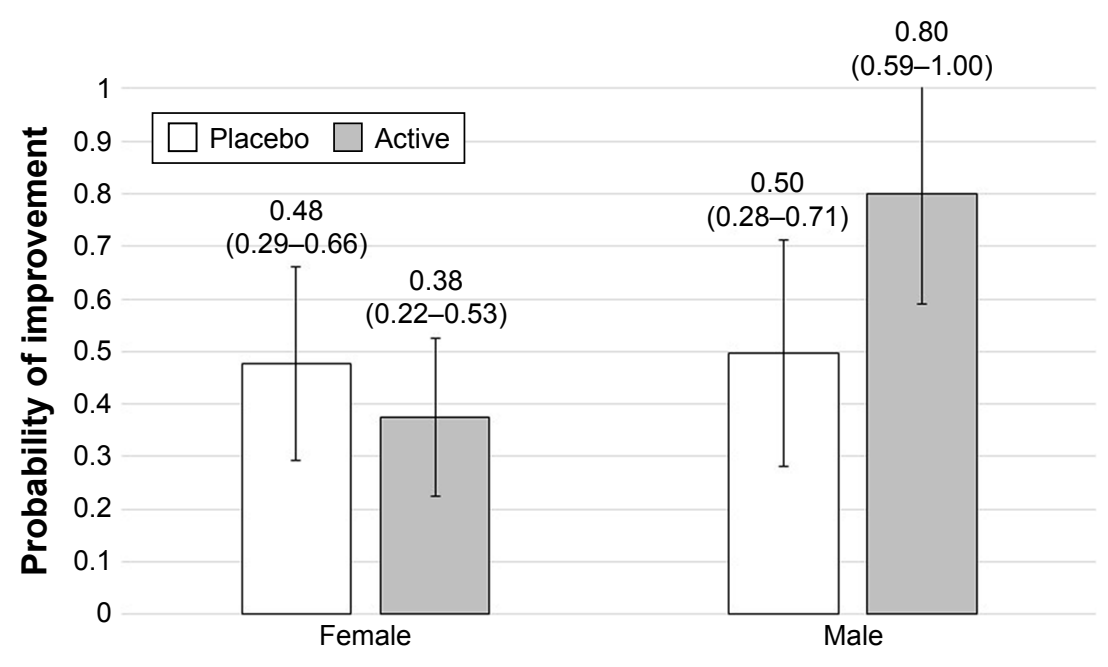

Figure 2 Improvement in MPI score at follow-up, according to treatments and gender. Abbreviation: MPI, Multidimensional Prognostic Index. 
Table 2 Change in primary (MPI) and component efficacy end points according to the treatment groups

\begin{tabular}{llll}
\hline Variable & Active & Placebo & $p$-value \\
\hline MPI & $-0.03(0.10)$ & $-0.04(0.1 \mathrm{I})$ & 0.367 \\
\hline SPMSQ & $-0.17(\mathrm{I} .43)$ & $-0.35(\mathrm{I} .98)$ & 0.563 \\
Exton-Smith scale & $+0.10(\mathrm{I} .98)$ & $+0.82(2.29)$ & 0.070 \\
ADL & $+0.10(\mathrm{I} .20)$ & $+0.39(\mathrm{I} .39)$ & 0.235 \\
IADL & $-0.27(\mathrm{I} .30)$ & $0.00(\mathrm{I} .04)$ & 0.194 \\
CIRS & $0.00(\mathrm{I} .40)$ & $+0.04(\mathrm{I} .25)$ & 0.887 \\
MNA & $+\mathrm{I} .0 \mathrm{I}(4.03)$ & $+0.92(3.29)$ & 0.894 \\
Number of drugs & $-0.18(2.01)$ & $-0.23(2.07)$ & 0.906 \\
\hline
\end{tabular}

Note: Data are presented as mean ( \pm standard deviation).

Abbreviations: $A D L$, activities of daily living; CIRS, cumulative illness rating scale; IADL, instrumental activities of daily living; MNA, mini nutritional assessment; MPI, Multidimensional Prognostic Index; SPMSQ, Short Portable Mental State Questionnaire.

and loss of function. ${ }^{17}$ Oral amino acid supplementation is considered an effective intervention to prevent or attenuate the loss of muscle mass and strength associated with inactivity and reduced intake of nutrients. ${ }^{18,19}$ Furthermore, it has been suggested that amino acid supplementation might counteract other negative multisystemic biological pathways, including insulin resistance, systemic inflammation, and enhanced oxidative stress, that are often activated in older acute patients. ${ }^{20}$

This study investigated the effect of adding a specialized dietetic aliment with $12 \mathrm{~g}$ of amino acids to standard of care on change in global health status as measured by the MPI score. One month after treatment, the MPI score significantly decreased in the overall sample without significant differences in both study groups. This finding suggests that the overall improvement of the health status of the study population over time is independent of AG treatment. Nevertheless, in the analysis stratified according to gender, we found that men allocated in the experimental group had a significantly higher probability of MPI improvement compared to the counterpart allocated to placebo, whereas no difference was detected in women.

These findings are in line with the results of a recent meta-analysis of trials conducted on medical inpatients that did not show any significant effect of nutritional supplementation on mortality, inhospital infection, and functional status. ${ }^{14}$ Similarly, a previous Cochrane systematic review and meta-analysis ${ }^{21}$ failed to demonstrate a clear significant benefit of protein and energy supplementation in terms of functional status in older people at risk of malnutrition. A more recent study, not included in the abovementioned meta-analyses, ${ }^{22}$ demonstrated that among acutely ill malnourished older patients, oral nutrition and caloric supplementation including known essential macro- and micronutrients, high protein composition supplemented with beta-hydroxy beta-methylbutyrate decreased mortality

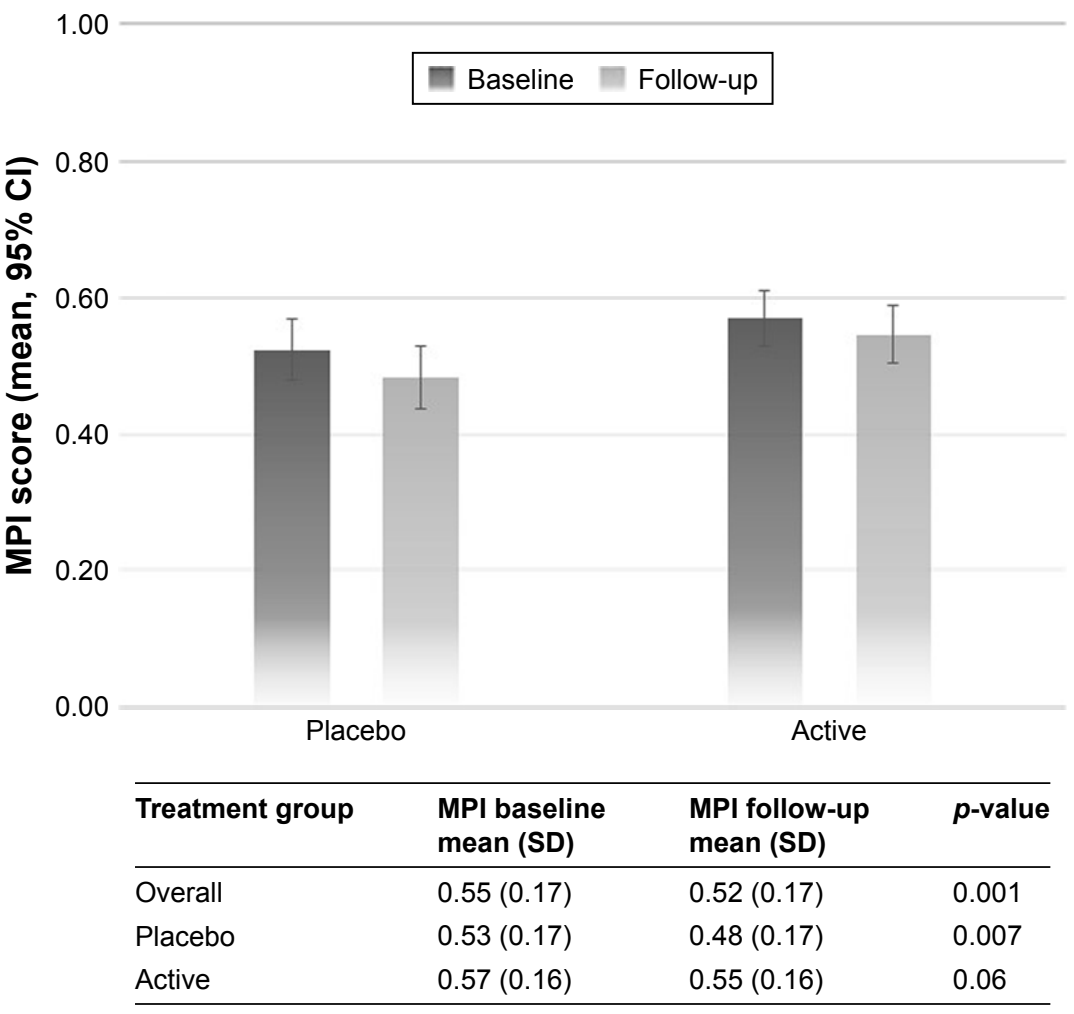

Figure 3 Likelihood of MPI improvement at follow-up according to the treatment group and gender.

Abbreviations: $\mathrm{Cl}$, confidence interval; MPI, Multidimensional Prognostic Index. 
and improved indices of nutritional status during the 90-day observation period, but no information was collected on functional and overall health status. Conversely, when oral nutritional supplementation has been combined with physical exercise program, a significant benefit in terms of muscle mass, muscle strength, physical performance, and quality of life has been demonstrated in both frail institutionalized older people $\mathrm{e}^{23}$ and sarcopenic patients enrolled in rehabilitation programs. ${ }^{24}$

Only a few studies specifically investigated the effect of oral amino acid supplementation using a composite indicator of global health status like the MPI. Indeed, most of the previous studies investigated single specific aspects of health status including muscle mass, grip strength, physical performance, ADL disability, and quality of life. MPI summarizes in a single score the status of several domains of health of older people including functional and cognitive status, nutritional status, multimorbidity, polypharmacy, and social status. Impairment of these domains has been recognized as key determinant of frailty of older people. Globally taken, the results of our study did not confirm the hypothesis that oral amino acid supplementation for a period of 4 weeks significantly reduces the frailty status and overall health condition in acutely ill patients after being discharged from the hospital. However, in line with previous studies that demonstrated a greater response to amino acid supplementation in men compared to women, ${ }^{25,26}$ this study suggests that oral amino acid supplementation might be more effective in men compared to women.

MPI might not be sensitive enough to capture the effect of amino acid supplementation. Although no effect was detected on any of the different domains assessed by MPI, we were not able to assess differences in other potential end points including muscle mass, strength, and function. A second potential explanation for the lack of difference in the primary outcome of the study might be a very high rate of MPI improvement during the follow-up. Indeed, in a previous observational study conducted in a large cohort of hospitalized older patients, we demonstrated a mean MPI change of -0.003 points between baseline and hospital discharge. ${ }^{27}$ Conversely, in this study, the average MPI change was -0.03 , which is 10 times higher compared to the previous observation; furthermore, MPI score significantly improved in both study groups reducing the likelihood of detecting a significant effect of the active intervention. Finally, 4 weeks of nutritional supplementation might not be sufficient to achieve a significant improvement of the overall health status of older frail patients.
This double-blind, randomized, placebo-controlled study showed that a specialized, dietetic aliment with $12 \mathrm{~g}$ amino acids did not alter the primary composite end point of MPI change after 30-day treatment period in an unselected sample of acutely ill older adults hospitalized for an acute medical problem. However, oral amino acid supplementation in addition to the standard care was associated with higher likelihood of MPI improvement among men. Further analyses are required to confirm and understand the biological mechanism(s) underlying these observed effects.

\section{Acknowledgments}

The abstract of this paper was presented at the XXXI Conference of the Società Italiana di Geriatria Ospedale Territorio as oral presentation with interim findings. The poster's abstract was published in "Abstract book" in Geriatric Care. 2017;3 (1S): https://doi.org/10.4081/gc.2017.6813.

\section{Disclosure}

The authors report no conflicts of interest in this work.

\section{References}

1. Creditor MC. Hazards of hospitalization of the elderly. Ann Intern Med. 1993;118:219-223.

2. Volpato S, Onder G, Cavalieri M, et al. Characteristics of nondisabled older patients developing new disability associated with medical illnesses and hospitalization. J Gen Intern Med. 2007;22:668-674.

3. Martone AM, Bianchi L, Abete P. The incidence of sarcopenia among hospitalised older patients. Results from the Glisten Study. J Cachexia Sarcopenia Muscle. 2017;8:907-914.

4. Sullivan DH, Sun S, Walls RC. Protein-energy undernutrition among elderly hospitalized patients: a prospective study. JAMA. 1999; 281:2013e9.

5. Solerte SB, Fioravanti M, Locatelli E, et al. Improvement of blood glucose control and insulin sensitivity during a long-term (60 weeks) randomized study with amino acid dietary supplements in elderly subjects with type 2 diabetes mellitus. Am J Cardiol. 2008;101:82E-88E.

6. Lim SL, Ong KC, Chan YH, Loke WC, Ferguson M, Daniels L. Malnutrition and its impact on cost of hospitalization, length of stay, readmission and 3-year mortality. Clin Nutr. 2012;31:345e50.

7. Bally MR, Blaser Yildirim PZ, Bounoure L, et al. Nutritional support and outcomes in malnourished medical inpatients: a systematic review and meta-analysis. JAMA Intern Med. 2016;176:43-53.

8. Pilotto A, Ferrucci L, Franceschi M, et al. Development and validation of a Multidimensional Prognostic Index for one-year mortality from comprehensive geriatric assessment in hospitalized older patients. Rejuvenation Res. 2008;11:151-161.

9. Volpato S, Bazzano S, Fontana A, Ferrucci L, Pilotto A; on behalf of the MPI-TriVeneto Study Group. Multidimensional prognostic index predicts mortality and length of stay during hospitalization in the older patients: a multicenter prospective study. J Gerontol A Biol Sci Med Sci. 2015;70:323-329.

10. Pilotto A, Sancarlo D, Pellegrini F, et al; on behalf of the FIRI-SIGG Study Group. The Multidimensional Prognostic Index predicts inhospital length of stay in older patients: a multicenter prospective study. Age Ageing. 2016;45:90-96.

11. Katz S, Downs TD, Cash HR, et al. Progress in the development of an index of ADL. Gerontologist. 1970;10:20-30. 
12. Lawton MP, Brody EM. Assessment of older people: self-maintaining and instrumental activities of daily living. Gerontologist. 1969;9: 179-186.

13. Pfeiffer E. A short portable mental status questionnaire for the assessment of organic brain deficit in elderly patients. J Am Geriatr Soc. 1975;23:433-441.

14. Linn B, Linn M, Gurel L. The cumulative illness rating scale. J Am Geriatr Soc. 1968;16:622-626.

15. Guigoz Y, Vellas B. The mini nutritional assessment (MNA) for grading the nutritional state of elderly patients: presentation of the MNA, history and validation. Nestle Nutr Workshop Ser Clin Perform Progr. 1999;1:3-11.

16. Bliss MR, McLaren R, Exton-Smith AN. Mattresses for preventing pressure sores in geriatric patients. Mon Bull Minist Health Public Health Lab Serv. 1966;25:238-268.

17. Bianchi L, Abete P, Bellelli G, et al; GLISTEN Group Investigators. Prevalence and clinical correlates of sarcopenia, identified according to the EWGSOP definition and diagnostic algorithm, in hospitalized older people: the GLISTEN study. J Gerontol A Biol Sci Med Sci. 2017; 72:1575-1581.

18. Ferrando AA, Paddon-Jones D, Hays NP, et al. EAA supplementation to increase nitrogen intake improves muscle function during bed rest in the elderly. Clin Nutr. 2010;29:18-23.

19. Deer RR, Goodlett SM, Fisher SR, et al. A randomized controlled Pilot trial of interventions to improve functional recovery after hospitalization in older adults: feasibility and adherence. J Gerontol A Biol Sci Med Sci. 2018;73:187-193.

20. Pasini E, Aquilani R, Dioguardi FS, D’Antona G, Gheorghiade M, Taegtmeyer H. Hypercatabolic syndrome: molecular basis and effects of nutritional supplements with amino acids. Am J Cardiol. 2008; 101:11E-15E.
21. Milne AC, Potter J, Vivanti A, Avenell A. Protein and energy supplementation in elderly people at risk from malnutrition. Cochrane Database Syst Rev. 2009;(2):CD003288.

22. Deutz NE, Matheson EM, Matarese LE, et al; NOURISH Study Group. Readmission and mortality in malnourished, older, hospitalized adults treated with a specialized oral nutritional supplement: a randomized clinical trial. Clin Nutr. 2016;35(1):18-26.

23. Abizanda P, López MD, García VP, et al. Effects of an oral nutritional supplementation plus physical exercise intervention on the physical function, nutritional status, and quality of life in frail institutionalized older adults: the ACTIVNES study. J Am Med Dir Assoc. 2015;16:439. e9-.e16.

24. Rondanelli M, Klersy C, Terracol G, et al. Whey protein, amino acids, and vitamin D supplementation with physical activity increases fat-free mass and strength, functionality, and quality of life and decreases inflammation in sarcopenic elderly. Am J Clin Nutr. 2016;103:830-840.

25. Smith GI, Atherton P, Villareal DT, et al. Differences in muscle protein synthesis and anabolic signaling in the postabsorptive state and in response to food in 65-80 year old men and women. PLoS One. 2008;3:e1875.

26. Alemán-Mateo H, Macías L, Esparza-Romero J, Astiazaran-García H, Blancas AL. Physiological effects beyond the significant gain in muscle mass in sarcopenic elderly men: evidence from a randomized clinical trial using a protein-rich food. Clin Interv Aging. 2012;7:225-234.

27. Volpato S, Daragjati J, Simonato M, Fontana A, Ferrucci L, Pilotto A. Change in the Multidimensional Prognostic Index Score during hospitalization in older patients. Rejuvenation Res. 2016;19:244-251. 


\section{Supplementary material}

Table SI Composition of one sachet of Aminoglutam ${ }^{\circledR}$

\begin{tabular}{ll}
\hline Components & Amount \\
\hline Amino acids & \\
L-Glutamine & $7,000 \mathrm{mg}$ \\
L-Leucine & $1,562.5 \mathrm{mg}$ \\
L-Lysine & $812 \mathrm{mg}$ \\
L-Isoleucine & $781.25 \mathrm{mg}$ \\
L-Valine & $781.25 \mathrm{mg}$ \\
L-Threonine & $437.5 \mathrm{mg}$ \\
L-Cysteine & $187.5 \mathrm{mg}$ \\
L-Histidine & $187.5 \mathrm{mg}$ \\
L-Phenylalanine & $125 \mathrm{mg}$ \\
L-Methionine & $62.5 \mathrm{mg}$ \\
L-Tyrosine & $37.5 \mathrm{mg}$ \\
L-Tryptophan & $25 \mathrm{mg}$ \\
Vitamins & \\
Vitamin C & $15 \mathrm{mg}$ \\
Vitamin B6 & $0.15 \mathrm{mg}$ \\
Vitamin BI & $0.15 \mathrm{mg}$ \\
Proteins & $0.0 \mathrm{mg}$ \\
Carbohydrates & $11.6 \mathrm{~g}$ \\
Fats & $0.18 \mathrm{~g}$ \\
Total energy & $96 \mathrm{kcal}$ \\
\hline
\end{tabular}

Clinical Interventions in Aging

Dovepress

\section{Publish your work in this journal}

Clinical Interventions in Aging is an international, peer-reviewed journal focusing on evidence-based reports on the value or lack thereof of treatments intended to prevent or delay the onset of maladaptive correlates of aging in human beings. This journal is indexed on PubMed Central, MedLine,

CAS, Scopus and the Elsevier Bibliographic databases. The manuscript management system is completely online and includes a very quick and fair peer-review system, which is all easy to use. Visit http://www.dovepress. com/testimonials.php to read real quotes from published authors. 